\title{
Sequential single-photon and direct two-photon absorption processes for Xe interacting with attosecond XUV pulses
}

\author{
A. Hadjipittas, ${ }^{1}$ H. I. B. Banks, ${ }^{1}$ B. Bergues,${ }^{2,3}$ and A. Emmanouilidou ${ }^{1}$ \\ ${ }^{1}$ Department of Physics and Astronomy, University College London, Gower Street, London WC1E 6BT, United Kingdom \\ ${ }^{2}$ Department of Physics, Ludwig-Maximilians-Universität Munich, Am Coulombwall 1, D-85748 Garching, Germany \\ ${ }^{3}$ Max Planck Institute of Quantum Optics, Hans-Kopfermann-Str. 1, D-85748 Garching, Germany
}

(Received 3 July 2020; accepted 11 September 2020; published 19 October 2020)

\begin{abstract}
We investigate the interaction of Xe with isolated attosecond XUV pulses. Specifically, we calculate the ion yields and determine the pathways leading to the formation of ionic charged states up to $\mathrm{Xe}^{5+}$. To do so, in our formulation we account for single-photon absorption, sequential multiphoton absorption, direct two-photon absorption, single and double Auger decays, and shake-off. We compare our results for the ion yields and for ion yield ratios with recent experimental results obtained for 93 and $115 \mathrm{eV}$ attosecond XUV pulses. In particular, we investigate the role that a sequence of two single-photon ionization processes plays in the formation of $\mathrm{Xe}^{4+}$. We find that each one of these two processes ionizes a core electron and thus leads to the formation of a double core-hole state. Remarkably, we find that the formation of $\mathrm{Xe}^{5+}$ involves a direct two-photon absorption process and the absorption of a total of three photons.
\end{abstract}

DOI: 10.1103/PhysRevA.102.043108

\section{INTRODUCTION}

The advent of free electron lasers (FELs) [1], has allowed for the production of ultrashort and high-energy laser pulses. These XUV pulses allow the ionization of inner-bound electrons that trigger a plethora of processes in atoms and molecules [2-4]. Xenon, with 54 electrons, is an ideal atom to investigate the effect that different ionization processes have on the formation of highly charged ionic states [5-11]. Previous studies have investigated the formation of $\mathrm{Xe}$ ion states up to $\mathrm{Xe}^{21+}[12-14]$ when a pulse of femtosecond duration at $93 \mathrm{eV}$ interacts with Xe. While FEL sources deliver high $\mathrm{XUV}$ pulse energies, the pulse duration is typically limited to the femtosecond range. In contrast, high-harmonic generation (HHG) based XUV sources can deliver isolated attosecond XUV pulses but the output pulse energy is limited by the low infrared to XUV conversion efficiency of the HHG process. This has prevented the observation of attosecond multiphoton interactions with inner-shell electrons for a long time. Such attosecond interactions were observed experimentally in Xe only recently [15]. The results of that study exhibited strong deviations with respect to sequential ionization via ionic ground states [15], which dominates the formation of lowercharged ionic states for femtosecond pulses [12,13]. Hence, the prevalent pathways for the formation of Xe ion charged states in the attosecond regime is still an open question.

Here, we address this question and model the interaction of Xe with an attosecond XUV pulse of energy 93 and $115 \mathrm{eV}$. The pulse parameters that we consider are chosen so that we can directly compare our results for ion yields up to $\mathrm{Xe}^{5+}$ and our results for ratios of the ion yields with the experimental ones obtained in Ref. [15]. Specifically, the pulses considered in Ref. [15] have photon energies of 93 and $115 \mathrm{eV}$ and a duration of about 340 as. Unlike previous studies [15], we account for sequential single-photon absorption processes via the creation of multiple core-hole states [16,17]. Moreover, we account for single-electron ionization by a two-photon absorption process, referred to as direct two-photon process $[13,18]$. This latter process has been found to affect the formation of ion charged states above $\mathrm{Xe}^{7+}$ in Ref. [13], where an XUV pulse of femtosecond duration is considered.

Pulses with photon energies of 93 and $115 \mathrm{eV}$ can access and ionize electrons from the $4 d$ subshell. The processes considered in our model include a single-electron ionization by single-photon absorption or by a direct two-photon absorption. In addition, we account for Auger decays [19]. In an Auger process, an electron falls from a higher-energy shell filling in an inner-shell hole. The energy released leads to the ionization of one or two bound electrons. We refer to the Auger decay as single or double depending on whether it leads to the ionization of one or two bound electrons, respectively. We also account for shake-off processes [20], resulting in the escape of a second electron following an ionization by a single-photon process.

In Sec. II, we describe the method that we use to investigate the interaction of Xe with an attosecond XUV pulse. In particular, we describe how to obtain the single-photon ionization cross sections and Auger decay rates that are involved in the rate equations $[12,21]$ that we employ. In Sec. III, we compute ion yields and yield ratios and compare them with experimental results [15]. In particular, we identify the main pathways leading to the formation of charged states up to $\mathrm{Xe}^{5+}$.

\section{METHOD}

We employ rate equations, as in Ref. [22] but with additional processes, in order to obtain the yields and pathways of the final ion states. In the rate equations we consider 
terms involving single-photon and two-photon ionization transitions, the Auger and double Auger decays as well as shake-off processes. The electronic configuration of $\mathrm{Xe}$ is $1 s^{2} 2 s^{2} 2 p^{6} 3 s^{2} 3 p^{6} 3 d^{10} 4 s^{2} 4 p^{6} 4 d^{10} 5 s^{2} 5 p^{6}$. A shell is distinguished by the $n$ quantum number and a subshell by the $n, l$ quantum numbers. A subshell is made up of $2 l+1$ orbitals, where each orbital has an occupancy of zero, one, or two electrons. In each $n p$ subshell we consider the orbitals $n p_{x}$, $n p_{y}$, and $n p_{z}$, and in each $n d$ subshell we consider the orbitals $n d_{x y}, n d_{y z}, n d_{x z}, n d_{x^{2}-y^{2}}$, and $n d_{z^{2}}$.

\section{A. Bound and continuum orbitals}

We denote the bound orbital wave function as $\phi_{i}$ and the continuum orbital wave function as $\phi_{\epsilon, l^{\prime}, m^{\prime}}$. To calculate the bound orbital wave functions, we use the molecular computing package MOLPRO [23] with the augmented quadruple-zeta plus polarization (AQZP) basis set [24]. This basis set expresses the orbitals as a combination of $l, m$ quantum numbers, whereas in our previous studies of Ar [4,22], each orbital was expressed by well-defined $l, m$ numbers and the $6-311 \mathrm{G}$ basis set was employed. Specifically, we express the bound orbital wave function as a product of a radial component and a spherical harmonic $Y_{l, m}(\theta, \phi)$ as follows:

$$
\phi_{i}(\mathbf{r})=\sum_{l, m} P_{i, l, m}(r) Y_{l, m}(\theta, \phi) / r
$$

To calculate the continuum wave function, we use the Herman-Skillman code $[25,26]$ to obtain the Hartree-FockSlater potential and the Numerov method [27] to obtain the radial part of the wave function, as was done in our previous works [22,28]. By multiplying the radial part with a spherical harmonic, the continuum wave function is given as

$$
\phi_{\epsilon, l^{\prime}, m^{\prime}}(\mathbf{r})=P_{\epsilon, l^{\prime}}(r) Y_{l^{\prime}, m^{\prime}}(\theta, \phi) / r
$$

By expressing the bound and continuum orbitals as a product of a radial and an angular component [see Eqs. (1) and (2)], we significantly simplify the evaluation of the singlephoton ionization cross sections and the Auger rates (see Secs. II B and II D).

\section{B. Single-photon ionization cross sections}

In order to calculate the photoionization cross section for an electron to transition from the bound orbital $\phi_{i}$ to the continuum orbital $\phi_{\epsilon, l^{\prime}, m^{\prime}}$, we use the equation below [29]:

$$
\sigma_{i \rightarrow \epsilon, l^{\prime}, m^{\prime}}=\frac{4}{3} \alpha \pi^{2} \omega N_{i} \sum_{M=-1,0,1}\left|D_{i \rightarrow \epsilon, l^{\prime}, m^{\prime}}^{M}\right|^{2},
$$

where $\alpha$ is the fine-structure constant, $N_{i}$ is the number of electrons in the initial orbital $i, \omega$ is the photon energy, and $M$ is the polarization of the photon. The matrix element $D_{i \rightarrow \epsilon, l^{\prime}, m^{\prime}}^{M}$ is given by

$$
D_{i \rightarrow \epsilon, l^{\prime}, m^{\prime}}^{M}=\int \phi_{i}(\mathbf{r}) \phi_{\epsilon, l^{\prime}, m^{\prime}}^{*}(\mathbf{r}) \sqrt{\frac{4 \pi}{3}} r Y_{1 M}(\theta, \phi) d \mathbf{r} .
$$

TABLE I. Single-photon ionization cross sections of neutral Xe interacting with a pulse of $93 \mathrm{eV}$ photon energy. The units of the cross sections are $\mathrm{cm}^{2}$.

\begin{tabular}{lcccc}
\hline \hline & Ref. [31] & Ref. [32] & Ref. [33] & This work \\
\hline$\sigma_{n=5}$ & $1.64 \times 10^{-18}$ & $1.47 \times 10^{-18}$ & $3.61 \times 10^{-19}$ & $3.30 \times 10^{-19}$ \\
$\sigma_{4 d^{10}}$ & & & $1.71 \times 10^{-17}$ & $2.24 \times 10^{-17}$ \\
\hline \hline
\end{tabular}

By subtituting in Eq. (4) the expansion for the bound and continuum orbitals from Eq. (1), we obtain the following:

$$
\begin{aligned}
D_{i \rightarrow \epsilon, l^{\prime}, m^{\prime}}^{M}= & \sqrt{\frac{4 \pi}{3}} \sum_{l m} \int_{0}^{\infty} d r P_{i, l, m}(r) r P_{\epsilon, l^{\prime}}(r) \\
& \times \int d \Omega Y_{l, m}(\theta, \phi) Y_{l^{\prime}, m^{\prime}}^{*}(\theta, \phi) Y_{1 M}(\theta, \phi) .
\end{aligned}
$$

Next, we calculate the angular integrals in terms of the Wigner-3 $j$ symbols [30] and obtain

$$
\begin{aligned}
D_{i \rightarrow \epsilon, l^{\prime}, m^{\prime}}^{M}= & \sum_{l m}(-1)^{m^{\prime}} \sqrt{(2 l+1)\left(2 l^{\prime}+1\right)} \\
& \times\left(\begin{array}{ccc}
l^{\prime} & l & 1 \\
0 & 0 & 0
\end{array}\right)\left(\begin{array}{ccc}
l^{\prime} & l & 1 \\
-m^{\prime} & m & M
\end{array}\right) \\
& \times \int_{0}^{\infty} d r P_{i, l, m}(r) r P_{\epsilon, l^{\prime}}(r) .
\end{aligned}
$$

Since only the energy of the final continuum orbital is of relevance, we sum in Eq. (3) over all $l^{\prime}$ and $m^{\prime}$ numbers to obtain

$$
\sigma_{i \rightarrow \epsilon}=\frac{4}{3} \alpha \pi^{2} \omega N_{i} \sum_{l^{\prime}, m^{\prime}} \sum_{M=-1,0,1}\left|D_{i \rightarrow \epsilon, l^{\prime}, m^{\prime}}^{M}\right|^{2} .
$$

In our calculations, the electronic configurations of $\mathrm{Xe}$ involved in the rate equations are expressed in terms of subshells. Hence, to find the single-photon ionization cross section from a certain subshell, we have to sum over all the cross sections involving the orbitals in this subshell. For instance, $\sigma_{2_{p}}=\sigma_{2_{p x}}+\sigma_{2_{p y}}+\sigma_{2_{p z}}$, where each of the $\sigma_{2_{p x}}, \sigma_{2_{p y}}$, and $\sigma_{2, z}$ are computed using Eq. (7).

In Table I, we compare our results with previous theoretical [33] and experimental [31,32] single-photon ionization cross sections.

We find that our computed cross sections for single-photon ionization from the $4 d$ subshell, $\sigma_{4 d^{10}}$, and from the $n=5$ shell, $\sigma_{n=5}$, are in very good agreement with the theoretical results in Ref. [33]. The difference between our work and Ref. [33] is that the latter employs the Hartree-Fock-Slater method to obtain both the bound and continuum orbitals, while we compute more accurately the bound orbitals using MOLPRO.

Moreover, we find that our cross section for ionization from the valence orbitals, $\sigma_{n=5}$, is roughly four times smaller than the one obtained experimentally $[31,32]$. This is in accord with Ref. [34] where it is explained that single-particle approximations lead to smaller computed valence cross sections compared to experimental ones. Since our valence cross sections differ from the experimental ones, we obtain results using our computed valence cross sections as well as using 
the experimental valence cross sections. We find that both sets of cross sections provide very similar results for the ion yields and the prevalent pathways. Therefore, in what follows, we present the results obtained using our computed valence ionization cross sections.

\section{Two-photon ionization cross sections}

Two-photon ionization involves a single-electron ionization following the simultaneous absorption of two photons. The two-photon ionization cross sections are computed via a method of scaling [35] and are the ones considered in Ref. [13]. For the long pulse considered in Ref. [13], two-photon ionization processes are included for transitions starting from Xe ion states with charge 5 and higher. For the short pulse employed in our work, we consider all twophoton ionization processes that are energetically allowed. However, if for a certain transition, both a single-photon and a two-photon ionization process are energetically allowed, we only account for the single-photon one. The reason is that the single-photon ionization cross section is roughly 30 orders of magnitude larger than the two-photon ionization cross section. Given the values for the two-photon ionization cross sections obtained in Ref. [13], we estimate that the two-photon ionization cross sections considered in our work vary between $10^{-48}$ and $10^{-47} \mathrm{~cm}^{4} \mathrm{~s}$. We obtain two different sets of results: one set using $10^{-47} \mathrm{~cm}^{4} \mathrm{~s}$ for all two-photon ionization cross sections and one using $10^{-48} \mathrm{~cm}^{4} \mathrm{~s}$. We find that both cross-section values result in similar pathways. However, the value of $10^{-47} \mathrm{~cm}^{4} \mathrm{~s}$ for the two-photon ionization cross sections leads to a better agreement with the experimental results for $\mathrm{Xe}^{5+}$. Thus, the results presented in Sec. III are for a two-photon ionization cross section of $10^{-47} \mathrm{~cm}^{4} \mathrm{~s}$.

\section{Auger decay}

The Auger rate is defined as follows [36]:

$$
\Gamma=\bar{\sum} 2 \pi|\mathcal{M}|^{2} \equiv \bar{\sum} 2 \pi\left|\left\langle\Psi_{f i}\left|H_{I}\right| \Psi_{i n}\right\rangle\right|^{2},
$$

where $\bar{\sum}$ means a summation over final states and an average over the initial states. The operator $H_{I}$ describes the Coulomb repulsion between the two electrons involved in the Auger transition. The derivation of the Auger decay rate for molecules in our previous work [37] involves bound molecular orbitals which are expressed as a sum of $l, m$ quantum numbers. In contrast, our previous work regarding the interaction of free-electron laser pulses with $\operatorname{Ar}[4,22]$ involves bound orbitals, where only one $l$ quantum number is associated with each orbital. Since, for Xe we consider bound orbitals which are expressed as a sum of $l, m$ quantum numbers, we adapt our formulation of the Auger process for molecules to atoms. As a result, we find that the matrix element for the Auger rate involving two valence orbitals $a$ and $b$, an inner-shell orbital $c$, and a continuum orbital $\epsilon$ with quantum numbers $l^{\prime}, m^{\prime}$ to be

$$
\begin{aligned}
\mathcal{M}= & \delta_{S^{\prime}, S} \delta_{M^{\prime}, M} \sum_{\substack{l_{c}, m_{c}, k \\
l_{a}, m_{a}, l_{b}, m_{b}}} \sum_{q=-k}^{k} \int d r_{1} \int d r_{2}(-1)^{m+m_{c}+q} \sqrt{\left(2 l^{\prime}+1\right)\left(2 l_{c}+1\right)\left(2 l_{b}+1\right)\left(2 l_{a}+1\right)} \\
& \times\left[P_{\epsilon, l^{\prime}}\left(r_{1}\right) P_{c, l_{c}, m_{c}}\left(r_{2}\right) \frac{r_{<}^{k}}{r_{>}^{k+1}} P_{b, l_{b}, m_{b}}\left(r_{1}\right) P_{a, l_{a}, m_{a}}\left(r_{2}\right)\left(\begin{array}{ccc}
l^{\prime} & k & l_{b} \\
0 & 0 & 0
\end{array}\right)\left(\begin{array}{cccc}
l^{\prime} & k & l_{b} \\
-m & -q & m_{b}
\end{array}\right)\left(\begin{array}{cccc}
l_{c} & k & l_{a} \\
0 & 0 & 0
\end{array}\right)\left(\begin{array}{cccc}
l_{c} & k & l_{a} \\
-m_{c} & q & m_{a}
\end{array}\right)\right. \\
& \left.+(-1)^{S} P_{\epsilon, l^{\prime}}\left(r_{1}\right) P_{c, l_{c}, m_{c}}\left(r_{2}\right) \frac{r_{<}^{k}}{r_{>}^{k+1}} P_{a, l_{a}, m_{a}}\left(r_{1}\right) P_{b, l_{b}, m_{b}}\left(r_{2}\right)\left(\begin{array}{ccccc}
l^{\prime} & k & l_{a} \\
0 & 0 & 0
\end{array}\right)\left(\begin{array}{ccccc}
l^{\prime} & k & l_{a} \\
-m & -q & m_{a}
\end{array}\right)\left(\begin{array}{cccc}
l_{c} & k & l_{b} \\
0 & 0 & 0
\end{array}\right)\left(\begin{array}{ccc}
l_{c} & k & l_{b} \\
-m_{c} & q & m_{b}
\end{array}\right)\right],
\end{aligned}
$$

where $r_{<}=\min \left(r_{1}, r_{2}\right)$ and $r_{>}=\max \left(r_{1}, r_{2}\right)$. The values $k$ and $q$ are the angular and magnetic quantum numbers of the spherical harmonics involved in the multipole expansion of the Coulomb interaction term $1 / r_{12} . S, S^{\prime}, M_{S}$, and $M_{S}^{\prime}$ are the initial and final total spins and the projection of these spins. The equation for the total Auger rate is given by Eq. (10),

$$
\Gamma_{b, a \rightarrow c}=\sum_{S, M_{S}, S^{\prime}, M_{S}^{\prime}} \pi N_{a b} N_{h} \sum_{\mathfrak{1}^{\prime}, m^{\prime}}|\mathcal{M}|^{2},
$$

where $N_{h}$ is the number of core holes in orbital $c$ and $N_{a b}$ is a normalization factor given by

$$
N_{a b}= \begin{cases}\frac{N_{a} N_{b}}{2 \times 2} & \text { different orbitals, } \\ \frac{N_{a}\left(N_{a}-1\right)}{2 \times 2 \times 1} & \text { same orbital, }\end{cases}
$$

where $N_{a}$ and $N_{b}$ denote the occupation numbers of orbitals $a$ and $b$. In order to obtain the Auger rate $\Gamma_{s, t \rightarrow u, \epsilon}$ between subshells $s, t$, and $u$, we add the Auger rates $\Gamma_{b, a \rightarrow c, \epsilon}$ over the $a$ and $b$ orbitals in the $s, t$ subshells. However, we do not sum over the $c$ orbitals in subshell $u$, since we average over the initial states.

\section{E. Double Auger decay}

The only energetically allowed double Auger decay process involves $\mathrm{Xe}^{+}$with a $4 d$ hole. In the double Auger process a $5 p$ electron drops in to fill the $4 d$ hole, while two more $5 p$ electrons escape to the continuum. According to Ref. [31] the double Auger decay rate is equal to $21 \%$ of the single Auger decay rate that involves the same initial state as the double Auger decay. The single Auger processes involve either a $5 p$ electron filling in the $4 d$ hole and the ionization of a $5 p$ electron or a $5 s$ electron filling in the $4 d$ hole while a $5 p$ or a 

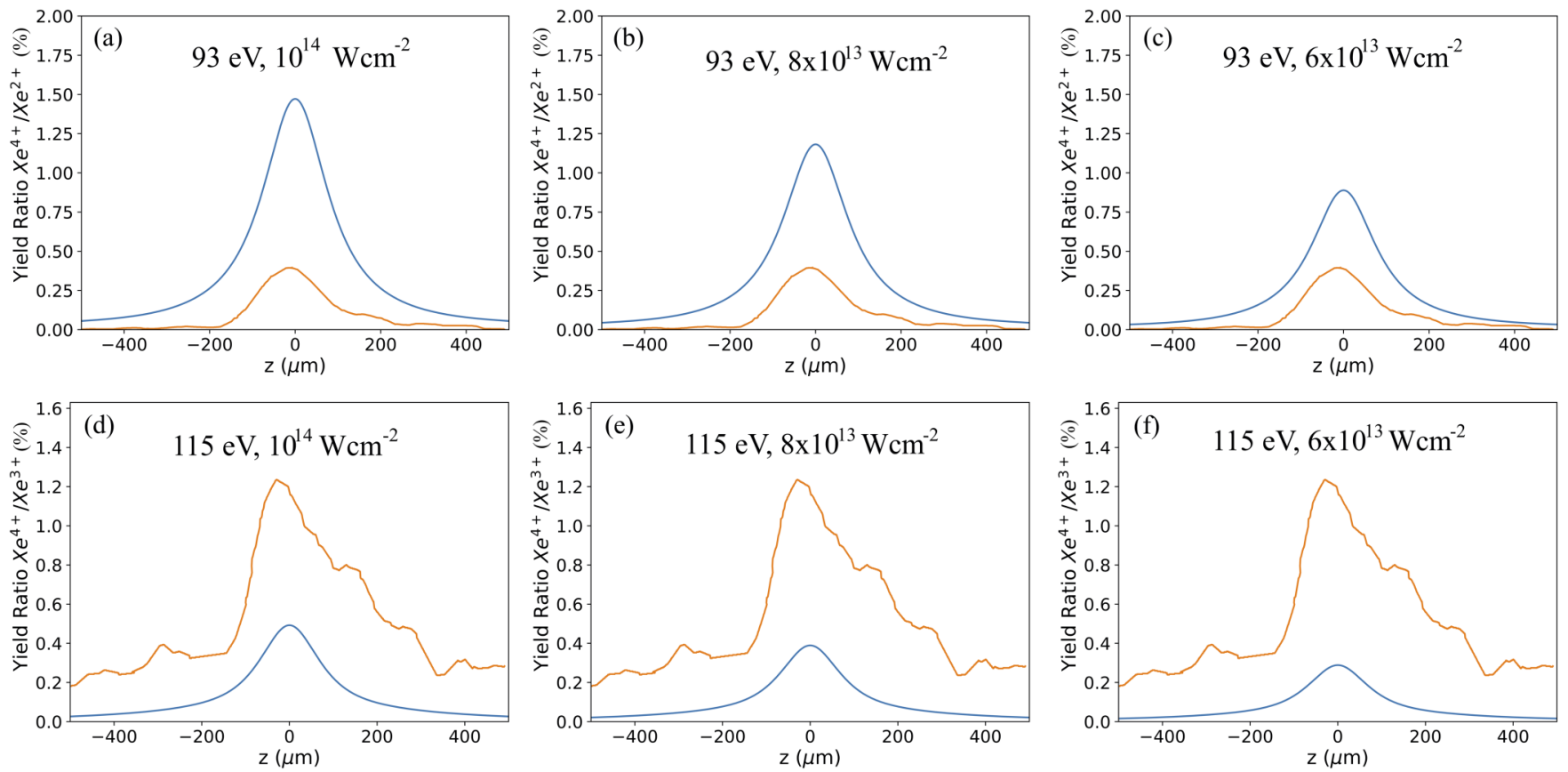

FIG. 1. Yield ratio as a function of the propagation distance in the focus. Orange lines (lighter gray) denote the experimental results adapted from Ref. [15] and blue lines (darker gray) denote our own ratios of the ion yields.

$5 s$ electron escapes. We find that the value of the double Auger decay rate is $6.14 \times 10^{-4}$ a.u.

\section{F. Shake-off}

When an electron escapes with high energy upon ionization there is a sudden change in the potential felt by the remaining bound electrons. This may cause a subsequent ionization of another bound electron, a process referred to as shake-off. Using the sudden approximation [38,39], we calculate the probability for an electron to be shaken off from the $n, l$ subshell as follows:

$$
P_{n l} \approx 1-\prod_{i=1}^{2 l+1}\left[\left|\int \phi_{i}^{*}\left(H_{i}\right) \phi_{i}\left(H_{f}\right) d \tau\right|^{2}\right]^{n_{i}},
$$

where $\phi_{i}^{*}\left(H_{i}\right)$ and $\phi_{i}\left(H_{f}\right)$ are the wave functions for the $2 l+1$ orbitals of the $n, l$ subshell in the initial and final Hamiltonians, respectively, and $n_{i}$ is the occupation of the $i$ orbital.

TABLE II. Relative ion yields and yield ratios for Xe interacting with an XUV pulse of photon energy $93 \mathrm{eV}$. The intensity is given in units of $\mathrm{W} \mathrm{cm}^{-2}$. The yields of all charged states add up to 100 .

\begin{tabular}{lccccc}
\hline \hline Ion & Ref. [15] & Ref. [32] & $10^{14}$ & $\begin{array}{c}\text { This work } \\
8 \times 10^{13}\end{array}$ & $6 \times 10^{13}$ \\
\hline $\mathrm{Xe}^{+}$ & 3.4 & 5.7 & 1.42 & 1.42 & 1.42 \\
$\mathrm{Xe}^{2+}$ & 77.6 & 68.6 & 74.8 & 74.8 & 74.8 \\
$\mathrm{Xe}^{3+}$ & 19.0 & 25.7 & 23.7 & 23.7 & 23.7 \\
$\mathrm{Xe}^{4+}$ & $4.0 \times 10^{-3}$ & & $1.5 \times 10^{-2}$ & $1.2 \times 10^{-2}$ & $8.9 \times 10^{-3}$ \\
\hline $\mathrm{Xe}^{2+}$ & & & & & \\
\hline \hline
\end{tabular}

\section{RESULTS}

Our goal is to identify the pathways leading to the formation of the charged states $\mathrm{Xe}^{4+}$ and $\mathrm{Xe}^{5+}$ for the pulse parameters used in the experiment described in Ref. [15]. These charged states are produced when Xe interacts with a pulse of full-width half-maximum of 340 as and photon energies of 93 and $115 \mathrm{eV}$. The energies needed to sequentially ionize electrons from the $4 d$ shell are roughly equal to $70 \mathrm{eV}$ for the removal of the first electron, $87 \mathrm{eV}$ for the removal of the second one, and $106 \mathrm{eV}$ for the removal of the third electron.

We employ a Gaussian laser pulse described in cylindrical coordinates as follows:

$$
I(r, z ; t)=I(t) \frac{w_{0}^{2}}{w(z)^{2}} \exp \left[\frac{-2 r^{2}}{w(z)^{2}}\right],
$$

where $r$ is the radius and $z$ is the beam propagation axis. The beam waist is denoted by $w_{0}$, which is equal to $0.85 \mu \mathrm{m}$ for the $93 \mathrm{eV}$ pulse and $2.12 \mu \mathrm{m}$ for the $115 \mathrm{eV}$ pulse. The beam

TABLE III. Relative ion yields and yield ratios for Xe interacting with an XUV pulse of photon energy $115 \mathrm{eV}$. The intensity is given in units of $\mathrm{W} \mathrm{cm}^{-2}$. The yields of all charged states add up to 100 .

\begin{tabular}{lccccc}
\hline \hline Ion & Ref. [15] & Ref. [32] & $10^{14}$ & $8 \times 10^{13}$ & $6 \times 10^{13}$ \\
\hline $\mathrm{Xe}^{+}$ & 2.95 & 7.23 & 7.23 & 7.23 \\
$\mathrm{Xe}^{2+}$ & 69.2 & 70.5 & 70.5 & 70.5 \\
$\mathrm{Xe}^{3+}$ & 27.8 & 22.3 & 22.3 & 22.3 \\
$\mathrm{Xe}^{4+}$ & $1.2 \times 10^{-2}$ & & $4.9 \times 10^{-3}$ & $3.9 \times 10^{-3}$ & $2.9 \times 10^{-3}$ \\
\hline $\mathrm{Xe}^{3+}$ & & & & \\
\hline \hline
\end{tabular}



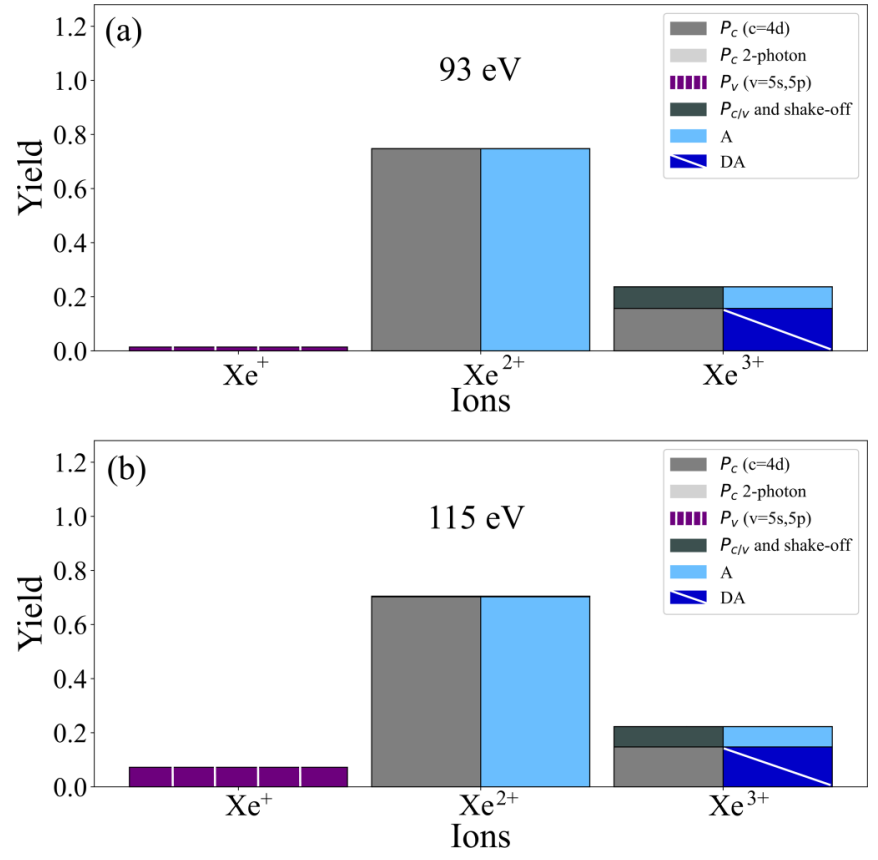

FIG. 2. Pathways leading to the formation of ion charges $\mathrm{Xe}^{+}$, $\mathrm{Xe}^{2+}$, and $\mathrm{Xe}^{3+}$ for two different photon energies of 93 and $115 \mathrm{eV}$. The vertical axis shows the yield of each ion state on the horizontal axis. The yields of all charged states add up to 1 . Each column corresponds to a different process. For each charged state, the sequence of the processes takes place from left to right. $P_{c}(c=4 d)$ stands for ionization of a $4 d$ electron by single-photon absorption; $P_{c}$ 2-photon stands for ionization of a $4 d$ electron by two-photon absorption; $P_{v}$ $(v=5 s, 5 p)$ stands for ionization of a valence electron $5 s$ or $5 p$ by single-photon absorption; $P_{c / v}$ and shake-off stands for ionization of a core or valence electron by single-photon absorption followed by ionization of another electron due to shake-off; A and DA stand for Auger decay and double Auger decay, respectively. The intensity considered is $10^{14} \mathrm{~W} \mathrm{~cm}^{-2}$.

radius at a distance $z$ is given below:

$$
w(z)=w_{0} \sqrt{1+\left(z / z_{R}\right)^{2}},
$$

where $z_{R}$ is the Rayleigh length and is equal to $93 \mu \mathrm{m}$ for both pulses. Furthermore, to calculate the ion yields and the prevalent pathways, we perform a volume averaging. To do so we consider a grid $(r, z)$ consisting of equidistant points. Namely, $r$ varies from 0 to $4.82 \mu \mathrm{m}$ in steps of $0.01 \mu \mathrm{m}$ and $z$ varies from $-10^{4}$ to $10^{4} \mu \mathrm{m}$ in steps of $0.5 \mu \mathrm{m}$. These grid points were chosen so that we obtain good convergence for the ion yields. At each grid point we compute the intensity of the pulse in accordance with Eq. (13). The ion yields are then calculated for each grid point. The sum of the respective yields of all grid points give us the total yield for each ion state.

\section{A. Ion yields and ratios of ion yields}

In what follows, we first compare our results with experimental ones for relative ion yields [15,32] and ion yield ratios [15]. In Ref. [15], the experimental pulse was obtained by high-harmonic generation while Ref. [32] involves synchrotron radiation. To account for the uncertainty in the intensity of the experimental results, we consider intensities

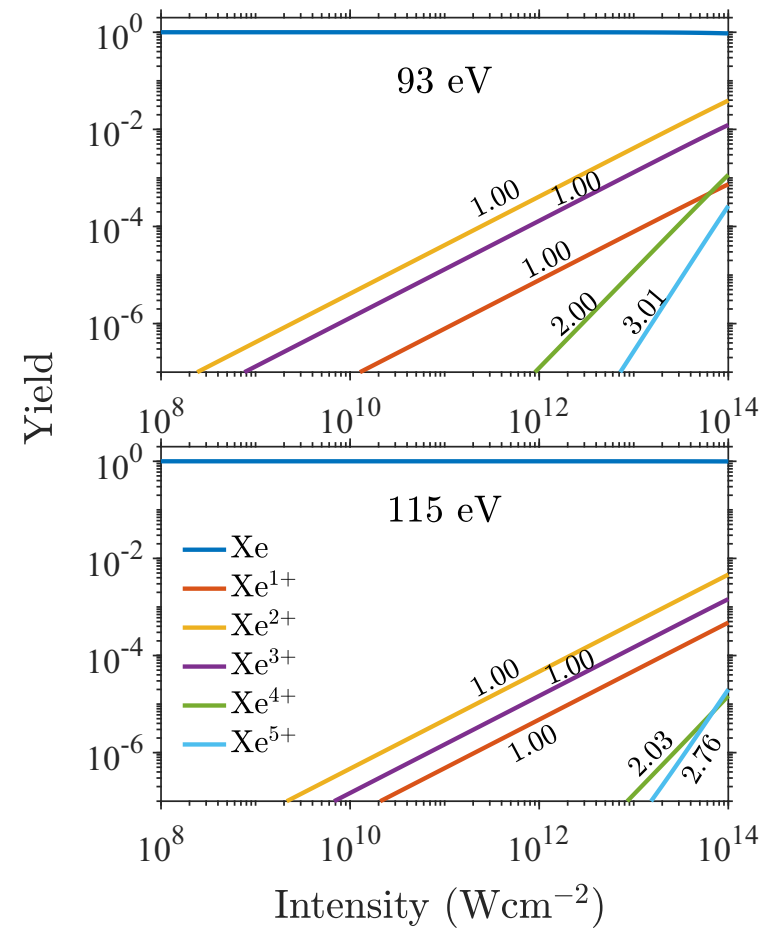

FIG. 3. Log-log plot of the ion yields versus pulse intensity for the $93 \mathrm{eV}$ pulse (a) and for the $115 \mathrm{eV}$ pulse (b). The numbers along the lines correspond to the slope of each of the yields versus intensity.

equal to $10^{14}, 8 \times 10^{13}$, and $6 \times 10^{13} \mathrm{~W} \mathrm{~cm}^{-2}$. Tables II and III show that our results for ion charges up to $\mathrm{Xe}^{3+}$ are in reasonable agreement with the experimental results for the charged states $\mathrm{Xe}^{2+}$ and $\mathrm{Xe}^{3+}$.

In Table II, we also show the ratio of the $\mathrm{Xe}^{4+}$ and $\mathrm{Xe}^{2+}$ ion yields for the $93 \mathrm{eV}$ pulse. We find that the difference with the experimental ratio in Ref. [15] depends on the intensity considered and roughly amounts to a factor of 2 for $6 \times 10^{13} \mathrm{~W} \mathrm{~cm}^{-2}$. Moreover, in Table III we compare the ratio of the ion yields $\mathrm{Xe}^{4+}$ and $\mathrm{Xe}^{3+}$ with the experimental ratio [15] for the $115 \mathrm{eV}$ pulse. We find that the ratio we compute differs by roughly a factor of 2 from the experimental result for $10^{14} \mathrm{~W} \mathrm{~cm}^{-2}$. We note that the ion yields for $\mathrm{Xe}^{4+}$ and $\mathrm{Xe}^{5+}$ are subjected to an experimental statistical uncertainty of up to $15 \%$. The deviations between the computed and the experimental values for the above ratios of the ion yields may be also partially explained by the experimental uncertainty in the pulse duration and intensity. In addition, in Fig. 1 we plot the dependence on the propagation axis $z$ of the ratio $\mathrm{Xe}^{4+} / \mathrm{Xe}^{2+}$ for the $93 \mathrm{eV}$ pulse and of the ratio $\mathrm{Xe}^{4+} / \mathrm{Xe}^{3+}$ for the $115 \mathrm{eV}$ pulse, for three different intensities. We believe that the agreement between theory and experiment within a factor of 2 is reasonable in view of the experimental uncertainties.

\section{B. Pathways}

Next, we identify the prevalent pathways that lead to the formation of $\mathrm{Xe}$ ion states $\mathrm{Xe}^{n+}$, where $n=1,2,3$. These pathways are shown in Fig. 2(a) for the $93 \mathrm{eV}$ pulse and in Fig. 2(b) for the $115 \mathrm{eV}$ pulse. In Fig. 2 the vertical axis 

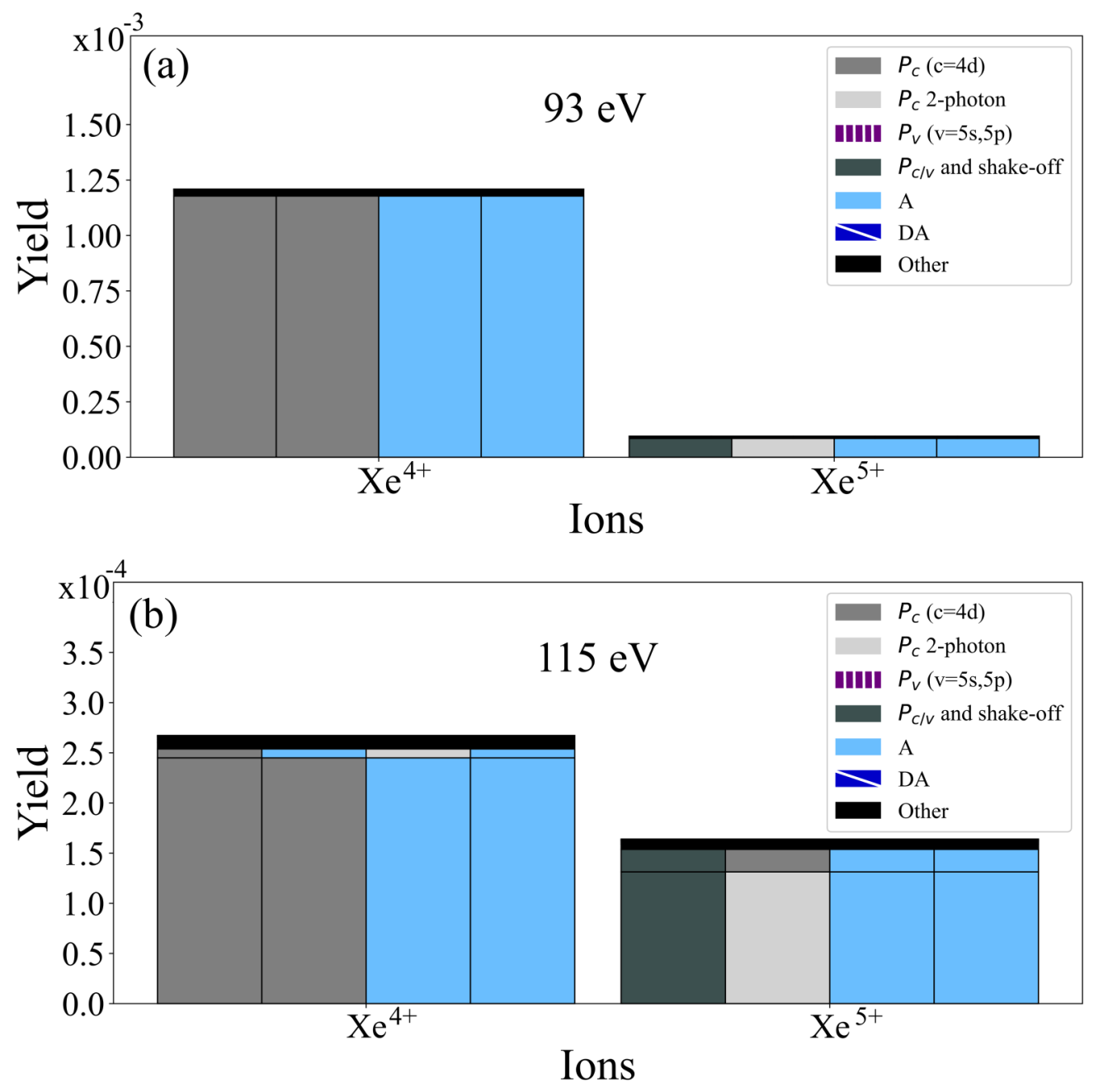

FIG. 4. Same as Fig. 2 for charged states $\mathrm{Xe}^{4+}$ and $\mathrm{Xe}^{5+}$.

corresponds to the relative ion yield of each ion state, where the latter is shown on the horizontal axis. The sum of the yields of all charged states of $\mathrm{Xe}^{n+}$, with $n=1-5$, is equal to 1. Figs. 2(a) and 2(b) show that the prevalent pathway leading to the formation of $\mathrm{Xe}^{+}$is ionization of a valence electron by single-photon absorption $\left[P_{v}(v=5 s, 5 p)\right]$. We also find that $\mathrm{Xe}^{2+}$ is formed by a sequence of two processes. The first process involves ionization of a core electron by single-photon absorption $\left[P_{c}(c=4 d)\right]$. The subsequent process is a single Auger decay $(A)$. In addition, we find that $\mathrm{Xe}^{3+}$ is formed mainly by ionization of a core electron by single-photon absorption $\left[P_{c}(c=4 d)\right]$ followed by a double Auger process (DA), i.e., an electron fills in the $4 d$ core hole, while two other electrons escape. Hence, $\mathrm{Xe}^{3+}$ is formed by a sequence of a single-photon absorption process and a double Auger one. As expected, our results for the prevalent pathways leading to the formation of $\mathrm{Xe}^{+}, \mathrm{Xe}^{2+}$, and $\mathrm{Xe}^{3+}$ are consistent with a slope equal to one on a log-log scale of the ion yields as a function of intensity [32] (see Fig. 3). As is well known, in the perturbative regime, the yield for an $N$-photon process, is proportional to $I^{N}$, where $I$ is the intensity.

In Fig. 4(a) for the $93 \mathrm{eV}$ pulse and Fig. 4(b) for the $115 \mathrm{eV}$ pulse, we show the prevalent pathways for charged states $\mathrm{Xe}^{4+}$ and $\mathrm{Xe}^{5+}$. We find that the prevalent pathway leading to the formation of $\mathrm{Xe}^{4+}$ consists of a sequence of four processes. First, a $4 d$ core electron is ionized by a singlephoton absorption $\left[P_{c}(c=4 d)\right]$. Then, before the $\mathrm{Xe}^{+}$ion relaxes, another $4 d$ core electron is ionized via single-photon absorption. We note that the time associated with the $\mathrm{Xe}^{+}$ ion, with a $4 d$ core hole, relaxing to $\mathrm{Xe}^{2+}$, with two valence electrons missing, is at least roughly three times larger than the duration of the 340 as pulse currently under consideration. Thus, the first two electrons are ionized by two sequential single-photon absorption processes forming a double corehole state. This is a process that was not accounted for in Ref. [15]. The third and fourth electrons are ionized by a sequence of two single Auger processes. Therefore, we find that the prevalent pathway leading to the formation of $\mathrm{Xe}^{4+}$ involves the absorption of two photons. This is consistent with the slope of the yield versus intensity of $\mathrm{Xe}^{4+}$ being equal to two (see Fig. 3).

For both the 93 and the $115 \mathrm{eV}$ pulses, we find that $\mathrm{Xe}^{5+}$ is formed mainly by one pathway that involves four processes. The first two electrons are ionized by a single-photon absorption followed by shake-off $\left(P_{c / v}\right.$ and shake-off). Next, a direct two-photon ionization process takes place $\left(P_{c} 2\right.$-photon). That is, a $4 d$ core electron escapes by absorbing two photons. Following the two ionization processes, two Auger decays 
take place, one after the other, resulting in the emission of the fourth and the fifth electrons. It is quite interesting that $\mathrm{Xe}^{5+}$ is formed by a pathway involving a two-photon ionization process when Xe interacts with an attosecond XUV pulse. In previous studies of Xe interacting with a femtosecond XUV pulse, two-photon ionization processes were found to play a significant role only for ion states higher than $\mathrm{Xe}^{7+}[13]$. Energetically, two photons would suffice for the formation of $\mathrm{Xe}^{5+}$. Surprisingly, we find that $\mathrm{Xe}^{5+}$ is preferentially created via absorption of three photons. As expected, this is reflected in the slope being roughly equal to 3 for $\mathrm{Xe}^{5+}$ in Fig. 3.

\section{CONCLUSION}

We have identified the main pathways leading to the formation of charged states up to $\mathrm{Xe}^{5+}$ when it interacts with an attosecond XUV pulse. Both for $\mathrm{Xe}^{4+}$ and for $\mathrm{Xe}^{5+}$ we find that the main pathway for their formation proceeds via two sequential photoabsorption processes, i.e., via the formation of a double core-hole state. For $\mathrm{Xe}^{4+}$ these sequential photoionization processes each involve one photon. However, for $\mathrm{Xe}^{5+}$ one of the two sequential photoionization processes involves a direct two-photon absorption process. So far, such direct two-photon absorption was only identified for the formation of charged states higher than $\mathrm{Xe}^{7+}$ for interaction with femtosecond XUV pulses [13].

\section{ACKNOWLEDGMENTS}

We acknowledge the use of the Legion computational resources at UCL. This work was funded by the Leverhulme Trust Research Project Grant No. 2017-376. B.B. acknowledges fruitful discussions with $\mathrm{H}$. Schröder and is grateful for support from M. Kling.
[1] C. Pellegrini, Eur. Phys. J. H 37, 659 (2012).

[2] J. Marangos, Contemp. Phys. 52, 551 (2011).

[3] J. Ullrich, A. Rudenko, and R. Moshammer, Annu. Rev. Phys. Chem. 63, 635 (2012).

[4] A. O. G. Wallis, H. I. B. Banks, and A. Emmanouilidou, Phys. Rev. A 91, 063402 (2015).

[5] L.-W. Pi and A. F. Starace, Phys. Rev. A 82, 053414 (2010).

[6] V. Richardson, J. T. Costello, D. Cubaynes, S. Düsterer, J. Feldhaus, H. W. van der Hart, P. Juranić, W. B. Li, M. Meyer, M. Richter, A. A. Sorokin, and K. Tiedke, Phys. Rev. Lett. 105, 013001 (2010).

[7] M. Richter, M. Y. Amusia, S. V. Bobashev, T. Feigl, P. N. Juranić, M. Martins, A. A. Sorokin, and K. Tiedtke, Phys. Rev. Lett. 102, 163002 (2009).

[8] S.-K. Son and R. Santra, Phys. Rev. A 85, 063415 (2012).

[9] K. Toyota, S.-K. Son, and R. Santra, Phys. Rev. A 95, 043412 (2017).

[10] B. Rudek, S.-K. Son, L. Foucar, S. W. Epp, B. Erk, R. Hartmann, M. Adolph, R. Andritschke, A. Aquila, N. Berrah et al., Nat. Photonics 6, 858 (2012).

[11] Y.-J. Chen, S. Pabst, A. Karamatskou, and R. Santra, Phys. Rev. A 91, 032503 (2015).

[12] M. G. Makris, P. Lambropoulos, and A. Mihelič, Phys. Rev. Lett. 102, 033002 (2009).

[13] P. Lambropoulos, K. G. Papamihail, and P. Decleva, J. Phys. B: At., Mol. Opt. Phys. 44, 175402 (2011).

[14] A. A. Sorokin, S. V. Bobashev, T. Feigl, K. Tiedtke, H. Wabnitz, and M. Richter, Phys. Rev. Lett. 99, 213002 (2007).

[15] B. Bergues, D. E. Rivas, M. Weidman, A. A. Muschet, W. Helml, A. Guggenmos, V. Pervak, U. Kleineberg, G. Marcus, R. Kienberger et al., Optica 5, 237 (2018).

[16] L. S. Cederbaum, F. Tarantelli, A. Sgamellotti, and J. Schirmer, J. Chem. Phys. 85, 6513 (1986).

[17] M. Tashiro, M. Ehara, H. Fukuzawa, K. Ueda, C. Buth, N. V. Kryzhevoi, and L. S. Cederbaum, J. Chem. Phys. 132, 184302 (2010).

[18] P. Lambropoulos, G. M. Nikolopoulos, and K. G. Papamihail, Phys. Rev. A 83, 021407(R) (2011).
[19] E. H. S. Burhop and W. N. Asaad, Adv. At. Mol. Phys. 8, 163 (1972).

[20] D. N. Fittinghoff, P. R. Bolton, B. Chang, and K. C. Kulander, Phys. Rev. Lett. 69, 2642 (1992).

[21] N. Rohringer and R. Santra, Phys. Rev. A 76, 033416 (2007).

[22] A. O. G. Wallis, L. Lodi, and A. Emmanouilidou, Phys. Rev. A 89, 063417 (2014).

[23] H. J. Werner, P. J. Knowles, R. Lindh, F. R. Manby, M. Schütz et al., MOLPRO, a package of ab initio programs, 2010.

[24] L. Martins, F. de Souza, G. Ceolin, F. Jorge, R. de Berrêdo, and C. Campos, Comput. Theor. Chem. 1013, 62 (2013).

[25] F. Herman and S. Skillman, Atomic Structure Calculations (Prentice-Hall, Englewood Cliffs, NJ, 1963).

[26] M. D. Pauli, Herman-Skillman program, Hermes.phys. uwm.edu/projects/elecstruct/elecstruct.html.

[27] B. V. Noumerov, Mon. Not. R. Astron. Soc. 84, 592 (1924).

[28] Henry I. B. Banks, Antonis Hadjipittas, and Agapi Emmanouilidou, Eur. Phys. J. D 74, 98 (2020).

[29] J. J. Sakurai, Modern Quantum Mechanics (Addison-Wesley, Reading, MA, 1994).

[30] A. R. Edmonds, Angular Momentum in Quantum Mechanics (Princeton University Press, Princeton, NJ, 1960).

[31] U. Becker, D. Szostak, H. G. Kerkhoff, M. Kupsch, B. Langer, R. Wehlitz, A. Yagishita, and T. Hayaishi, Phys. Rev. A 39, 3902 (1989).

[32] D. M. P. Holland, K. Codling, G. V. Marr, and J. B. West, J. Phys. B 12, 2465 (1979).

[33] J. Yeh and I. Lindau, At. Data Nucl. Data Tables 32, 1 (1985).

[34] M. Y. Amusia, L. V. Chernysheva, and V. K. Ivanov, Phys. Lett. A 43, 243 (1973).

[35] P. Lambropoulos and X. Tang, J. Opt. Soc. Am. B 4, 821 (1987).

[36] W. Pauli, Wave Mechanics: Volume 5 of Pauli Lectures on Physics (Wiley, New York, 2000), pp. 150-151.

[37] H. I. B. Banks, D. A. Little, J. Tennyson, and A. Emmanouilidou, Phys. Chem. Chem. Phys. 19, 19794 (2017).

[38] T. Åberg, Ann. Acad. Sci. Fenn., Ser A VI 308, 1 (1969).

[39] T. A. Carlson and C. W. Nestor, Phys. Rev. A 8, 2887 (1973). 